Research Article

\title{
Gengnianchun Recipe Protects Ovarian Reserve of Rats Treated by 4-Vinylcyclohexene Diepoxide via the AKT Pathway
}

\author{
Fangui Zhao $\mathbb{D}^{1,2}$ and Wenjun Wang $\mathbb{D}^{2,3}$ \\ ${ }^{1}$ Department of Ultrasound Diagnosis, Obstetrics and Gynecology Hospital, Fudan University, Shanghai 200011, China \\ ${ }^{2}$ Shanghai Key Laboratory of Female Reproductive Endocrine Related Diseases, Shanghai 200011, China \\ ${ }^{3}$ Department of Integrated Traditional Chinese Medicine and Western Medicine, Obstetrics and Gynecology Hospital, \\ Fudan University, Shanghai 200011, China \\ Correspondence should be addressed to Wenjun Wang; wangwenjun985@fckyy.org.cn
}

Received 1 April 2020; Revised 16 November 2020; Accepted 1 December 2020; Published 16 December 2020

Academic Editor: Ma gorzata Kotula Balak

Copyright (C) 2020 Fangui Zhao and Wenjun Wang. This is an open access article distributed under the Creative Commons Attribution License, which permits unrestricted use, distribution, and reproduction in any medium, provided the original work is properly cited.

\begin{abstract}
Diminished ovarian reserve (DOR) refers to a decrease in the number and quality of oocytes. Western treatment of DOR does not improve the ovarian reserve fundamentally, and the effect is limited. Gengnianchun recipe (GNC) is a traditional Chinese medicine formula originally applied to treat menopausal syndrome but is also found to be effective in treating clinical DOR patients. Here we aim to examine the effect of GNC in a DOR rat model induced by 4-vinylcyclohexene diepoxide (VCD), a chemical that selectively destroys ovarian small preantral follicles, and further investigate the possible mechanisms. Female SD rats were randomly divided into four groups: control group (C), model group (M), high-dose GNC group (H), and low-dose GNC group (L). Rats in M, H, and L were administered with VCD and normal saline, high-dose GNC, and low-dose GNC separately. Rat ovaries were harvested either to conduct HE staining for follicle count, immunohistochemistry, or western blot. We found that high dose of GNC significantly increased the ovarian index and sustained the number of primordial follicles and primary follicles in VCD treated rats. Moreover, high dose of GNC significantly increased the ovarian protein expression of mouse vasa homologue $(\mathrm{MVH})$, anti-Müllerian hormone $(\mathrm{AMH})$, follicle-stimulating hormone receptor (FSHR), and estrogen receptor $\beta$ (ER $\beta$ ) compared with that in the model group. Besides, high-dose GNC significantly increased ovarian AKT phosphorylation and the expression of downstream forkhead box O3 (FOXO3a). Proapoptosis proteins of Bax, cleaved caspase-3, and poly ADP-ribose polymerase (PARP) were significantly decreased after high-dose GNC treatment compared with those in the model group. Taken together, these findings suggest that high-dose GNC could protect ovarian reserve against VCD-induced toxicity via the activation of the AKT signaling pathway and reduced cell apoptosis in SD Rats. This effect could either be induced by the increased FSHR signaling or by the nontranscriptional activation of $\mathrm{ER} \beta$, which requires further investigation.
\end{abstract}

\section{Introduction}

Diminished ovarian reserve (DOR) refers to a decrease in the number and quality of oocytes $[1,2]$. DOR patients may have irregular or regular menstruation but bear a lower chance of pregnancy and a higher chance of abortion. Different from the physiological ovarian reserve decline after menopause, DOR usually refers to the early or accelerated occurrence of ovarian reserve decline in women of reproductive age [3]. The etiology of DOR remains unclear. Genetic factors, environmental pollution, autoimmune diseases, psychological factors, and iatrogenic factors may be involved. Current consensus holds that it is a disease with complex causes and lack of treatment approaches with definitive efficacy.

Gengnianchun recipe (GNC) is an effective traditional Chinese formula applied in our hospital to treat postmenopausal syndrome for decades [4]. Our group has studied the effect of GNC on various animal models to reveal its mechanism in treating various aging-related diseases such as osteoporosis, Alzheimer's disease, immune dysfunction, and skin aging [5-13]. In traditional Chinese medicine theories, DOR shares a similar etiology with menopause, which is the 
deficiency in the kidney. Therefore, we have also applied GNC on clinical DOR patients and observed great effect in recovering ovarian reserve and improving pregnancy rate. But this has not been verified in the animal model and the mechanism remains unknown.

4 -vinylcyclohexene diepoxide (VCD) is an environmental chemical produced during the manufacture of rubber tires, flame retardants, insecticides, plasticizers, and antioxidants. Earlier studies have shown that short-term use of VCD can damage the primordial follicles and primary follicles of Sprague-Dawley (SD) rat ovaries to induce the initial stage of DOR $[14,15]$. Also, VCD might work through the inhibition of the PI3K-AKT signaling pathway to induce apoptosis and follicle atresia $[16,17]$. In this study, we aim to explore the effect of GNC on a VCD-induced DOR rat model and to further investigate its influence on PI3K-AKT pathway molecules to explore the underlying mechanism, thereby providing an experimental basis for the application of GNC in DOR therapy.

\section{Materials and Methods}

2.1. Animals and Treatments. A total of forty 3- to 4-weekold female SD rats weighing $35 \pm 3.5 \mathrm{~g}$ were purchased from Shanghai JieSiJie Laboratory Animal Co., Ltd., China. Rats were housed in the SPF facility with a standard pellet diet and water was provided ad libitum at a temperature of $22-25^{\circ} \mathrm{C}$ and relative humidity of $50-60 \%$ with daily 12 -hour light. After adaptation for 5 days, all rats were randomly divided into four groups: control group (C), model group (M), high-dose GNC group (H), and low-dose GNC group (L). Rats in group $\mathrm{M}, \mathrm{H}$, and $\mathrm{L}$ were given $\mathrm{VCD}$ (94956, Sigma-Aldrich, USA) by intraperitoneal injection on a dosage of $80 \mathrm{mg} / \mathrm{kg}$ bodyweight for 20 days according to previous reports $[15,18]$, while rats in group $C$ were administered the same volume of solvent (S3547, SigmaAldrich, USA). In the meantime, rats in the groups $\mathrm{M}, \mathrm{H}$, and $\mathrm{L}$ were given normal saline, high-dose $(5.54 \mathrm{~g} / \mathrm{kg}$ body weight), or low-dose ( $2.77 \mathrm{~g} / \mathrm{kg}$ body weight) of GNC decoction, respectively, by gavage for 30 days. The low dose was converted from the human dose according to a previous report [19]. High dose was twice as high as the low dose. The time length of GNC application was set according to the clinical application length on DOR patients, which is 6 menstruation cycles and equals 30 days on rats. The GNC decoction was prepared by dissolving herb granules into hot water [7]. Intraperitoneal injection and oral gavage volume were adjusted according to the bodyweight, which was measured every three days [13]. All animal experimental procedures were approved by the Animal Experimental Ethical Committee of Fudan University (no.2015-07FCKYY-WWJ-01).

2.2. Sample Collection. After 30 days of manipulation, rats were given vaginal smears and observed for the predominant cell type under a light microscopic to verify the estrous cycle stage [20]. Rats that were not at estrous stage were selected to be sacrificed. Other rats were continued with previous manipulation and sacrificed until vaginal smear results turned to other stages of estrous cycle. Whole blood was collected from the heart after anesthesia. The collected whole blood was centrifuged at $3000 \mathrm{rpm}$ for 10 minutes at $4^{\circ} \mathrm{C}$ to retrieve serum. Bilateral ovaries were collected and immediately weighed to calculate the ovarian index by the following formulation: ovarian index = ovarian wet weight $/$ body weight. Uteruses were also collected for morphology observation. The left ovaries were fixed in $4 \%$ paraformaldehyde solution for 24 hours and then embedded in paraffin for preparation of slides by Wuhan Servicebio Technology Co., Ltd. The right ovaries were dipped in liquid nitrogen and transferred to a- $80^{\circ} \mathrm{C}$ freezer until further use.

2.3. Ovary Serial Section and Follicle Counting. After the ovarian tissue was paraffin-embedded, the largest sections of ovaries were selected for continuous sectioning. Three sections at the 10th, 20th, and 30th sections of the largest sections of each ovary were selected to perform hematoxylin-eosin (HE) staining for histological observation and follicle count. Follicles were classified into various stages according to the modified Oktay system [21]. The analysis was repeated three times with each replicate containing 18 slices of 6 ovaries from 6 rats in each group.

2.4. Serum Hormone Assay. Serum estradiol $\left(E_{2}\right)$ and testosterone $(T)$ levels were detected by the chemiluminescence method. The commercially available kits were used to measure the serum concentration of $E_{2}$ (33540, Beckman, USA, detection range: $13-4096 \mathrm{pg} / \mathrm{mL}$ ) and $T$ (33550, Beckman, USA, detection range: $0.1-16 \mathrm{ng} / \mathrm{mL}$ ) by the Beckman Coulter Unicel DxI 800 system according to the manufacturer's protocols. The analysis was repeated three times with each replicate containing 6 samples from 6 rats in each group.

2.5. Immunohistochemistry. The ovarian tissue sections were dewaxed with xylene and ethanol. After the antigen retrieval, the sections were exposed to primary antibodies overnight at $4^{\circ} \mathrm{C}$. Antibody information has been listed in Table 1. The tissues were incubated with horseradish peroxidase-linked secondary antibodies for 40 minutes at room temperature and then stained with DAB solution and hematoxylin for visualization. After dehydration, the sections were sealed with neutral gum and observed under a microscope. The analysis was repeated three times with each replicate containing 6 slices of 6 ovaries from 6 rats in each group.

2.6. Western Blot. The right ovaries were dipped in the lysis buffer and homogenized by an ultrasound homogenizer. The lysate was then centrifuged at $12000 \mathrm{rpm}$ for 30 minutes at $4^{\circ} \mathrm{C}$. The supernatant was harvested and boiled with loading buffer for SDS-PAGE. Proteins were subjected to electrophoresis in SDS-polyacrylamide gels and transferred to polyvinylidene fluoride membranes. After blocking with nonfat milk, the membranes were exposed to primary antibodies overnight at $4^{\circ} \mathrm{C}$. Antibody information has also 
TABLE 1: The product information of the primary antibodies used in this study.

\begin{tabular}{|c|c|c|}
\hline Target & Catalog number and product source & $\begin{array}{l}\text { Application* (dilution } \\
\text { rate) }\end{array}$ \\
\hline DDX4/mouse vasa homolog (MVH) & ab13840, Abcam, USA & WB $(1: 1000) ;$ IHC $(1: 200)$ \\
\hline Anti-Müllerian hormone (AMH) & $\begin{array}{c}\text { LS-B4020, Lifespan Biological Sscience } \\
\text { Co., } \\
\text { USA }\end{array}$ & WB $(1: 1000) ;$ IHC $(1: 100)$ \\
\hline Follicle-stimulating hormone receptor (FSHR) & NBP1-46302, NOVUS, USA & WB $(1: 1000) ;$ IHC $(1: 250)$ \\
\hline Estrogen receptor $\alpha(\mathrm{ER} \alpha)$ & ab32063, Abcam, USA & WB $(1: 1000) ;$ IHC $(1: 200)$ \\
\hline Estrogen receptor beta $(\mathrm{ER} \beta)$ & ab3577, Abcam, USA & WB $(1: 1000) ;$ IHC $(1: 500)$ \\
\hline Forkhead box O3(FOXO3a) & \#12829, CST, USA & $\begin{array}{c}\text { WB }(1: 1000) ; \text { IHC }(1: \\
3000)\end{array}$ \\
\hline PolyADP-ribose polymerase (PARP) & \#9532, CST, USA & WB $(1: 1000)$ \\
\hline $\begin{array}{l}\text { Phosphatase and tensin homolog deleted on chromosome ten } \\
\text { (PTEN) }\end{array}$ & \#9188, CST, USA & WB $(1: 1000) ;$ IHC $(1: 125)$ \\
\hline Phospho-AKT (p-AKT, Ser473) & \#4060, CST, USA & WB $(1: 2000) ;$ IHC $(1: 100)$ \\
\hline $\mathrm{AKT}$ & \#2920, CST, USA & WB $(1: 2000) ;$ IHC $(1: 250)$ \\
\hline BAX & \#14796, CST, USA & WB $(1: 1000) ;$ IHC $(1: 400)$ \\
\hline $\mathrm{BAD}$ & \#9239, CST, USA & WB $(1: 1000)$ \\
\hline Cleaved caspase-3 (Asp175) & \#9664, CST, USA & $\begin{array}{l}\text { WB }(1: 1000) ; \text { IHC }(1: \\
2000)\end{array}$ \\
\hline
\end{tabular}

*WB: western blot; IHC: immunohistochemistry.

been listed in Table 1. Afterward, the membrane was incubated with antirabbit IgG HRP-linked antibodies (\#7074, CST, Inc., USA) for $1 \mathrm{~h}$ at room temperature and then visualized by the chemiluminescence method and observed under an ImageQuant LAS-4000 mini biomolecular imager, GE, USA. The relative protein expression levels were determined by Quantity One software. The analysis was repeated three times with each replicate containing 6 slices of 6 ovaries from 6 rats in each group.

\section{Statistical Methods}

The experimental data were presented as mean \pm standard deviation $(X \pm S D)$. SPSS18.0 was used for statistical analysis. The comparison of body weight changes between four groups was made using repeated measurement analysis of variance. ANOVA with the Bonferroni post hoc test was applied to test significances between treatment groups. $P<0.05$ was considered as a significant difference.

\section{Results}

4.1. General Condition of Rats and Observation of Reproductive Organs. To adjust the dose of VCD and GNC, body weight changes of rats in different groups were monitored every three days during 30-day manipulation. As Figure 1(a) shows, there was no significant difference between the groups during drug administration. This indicates that VCD and GNC do not affect the general health of rats. After 20 days of VCD administration, the ovarian index of the model group was significantly decreased compared to the control group (Figure $1(\mathrm{~b}), p<0.05$ ). After high-dose GNC treatment, the mean ovarian index seemed to increase a little than the group $M$. But the difference between the group $M$ and group $\mathrm{H}$ was not significant. Low dose of GNC did not affect the ovarian index. The appearance of the uterus and ovaries in the different groups is shown in Figure 1(c). We observed a lower volume of ovaries in the group $\mathrm{M}$ and $\mathrm{L}$ compared to that of the group $\mathrm{C}$ and $\mathrm{H}$. Also, there seemed to be less big antral follicles in the ovarian cortex in the group $\mathrm{M}$ and $\mathrm{L}$ than that of the group $\mathrm{C}$ and $\mathrm{H}$. Representative microscopic view of ovarian $\mathrm{HE}$ staining shows that there were plenty of primordial follicles accumulate at the ovarian cortex in the group $\mathrm{C}$ and $\mathrm{H}$, while the number of primordial follicles in the cortex in the group $M$ and $L$ was much lower (Figure 1(d)).

\subsection{Ovarian Reserve Changes after VCD and GNC Treatment.} As per the previous studies report, VCD mainly induced a significant decline in primordial and primary follicles compared to the control group (Figures 2(a) and 2(b), $P<0.05)$. High dose of GNC treatment significantly improved the number of primordial follicles and primary follicles (Figures 2(a) and 2(b), $P<0.05$ ). The mean number of primary follicles was even higher than the control group (Figure 2(b), $P<0.05$ ). There was no significant difference between the group $\mathrm{M}$ and $\mathrm{C}$ in secondary follicles, antral follicles, or corpus luteum (Figures 2(c)-2(e)). Also, there was no therapeutic effect of low-dose GNC observed in improving numbers of follicles at all stages or corpus luteum compared to the model group (Figures 2(a)-2(e)) Interestingly, high-dose of GNC significantly improved the antral follicle counts compared to both group $\mathrm{M}$ and group $\mathrm{C}$ (Figure 2(d), $P<0.05$ ).

\subsection{The Effect of VCD and GNC on the Reproductive Hormone} Level. $E_{2}$ and $T$ are two reproductive hormones secreted by granulosa cells and theca cells of follicles, respectively. Probably because VCD mainly causes the depletion of primordial follicles and primary follicles, and the number of both granulosa cells and theca cells are relatively small 


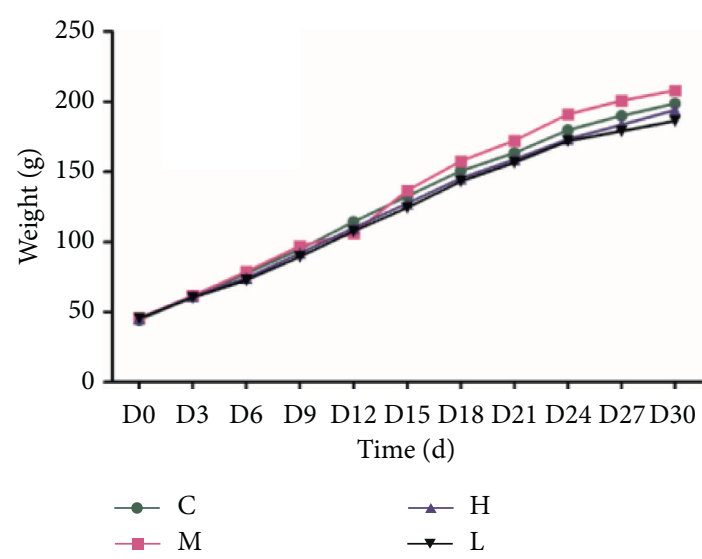

(a)

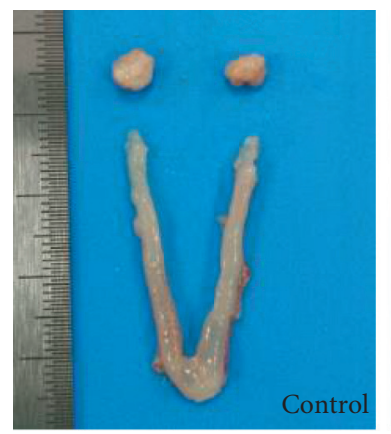

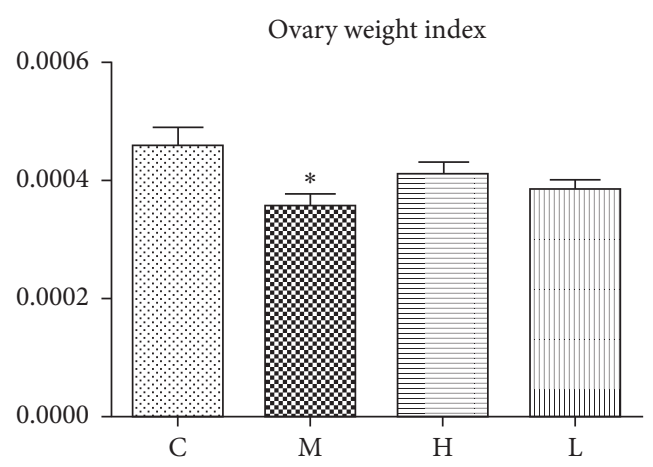

(b)
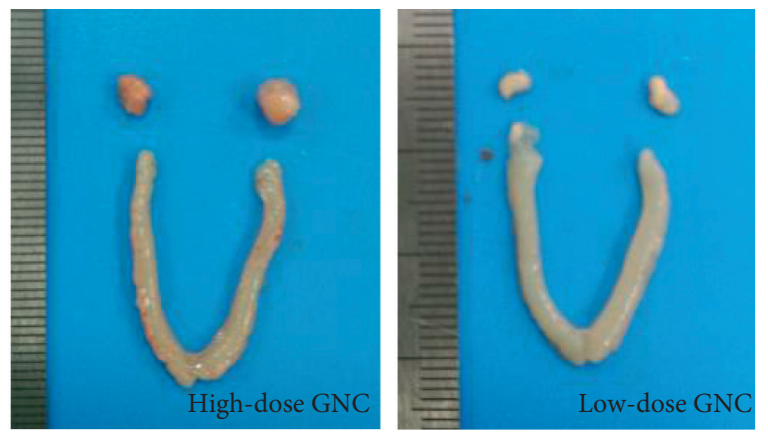

(c)
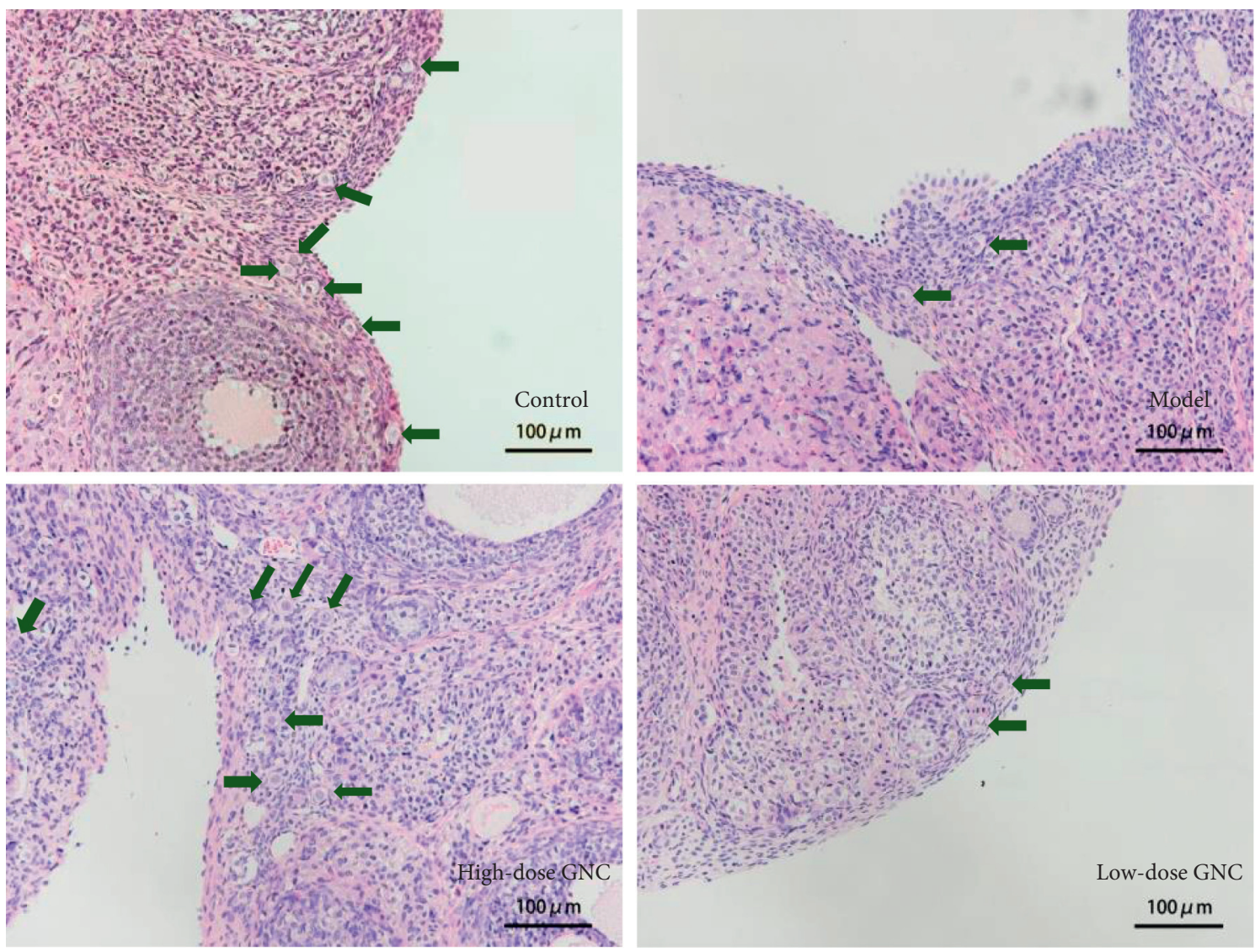

(d)

FIgURE 1: General conditions of rats and reproductive organs in different groups. (a) Bodyweight changes of rats in each group during experimental manipulation. (b) Ovarian weight index of rats in each group. (c) The representative appearance of ovaries and the uterus of rats in each group. (d) Representative view of HE staining of ovaries under a microscope. The brown arrows indicate primordial follicles. 


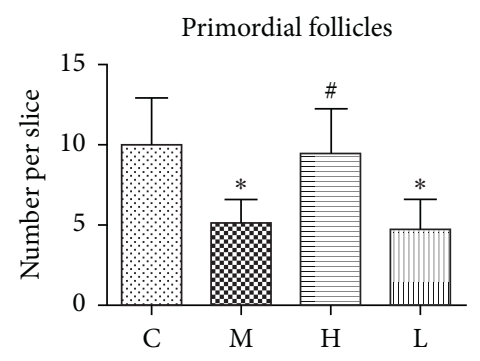

(a)

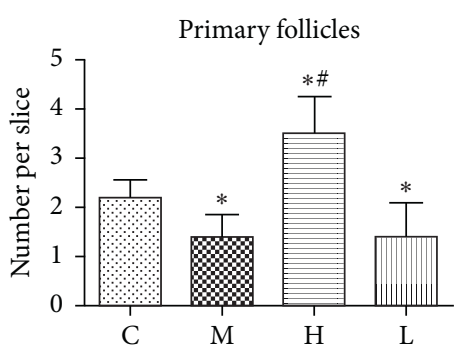

(b)

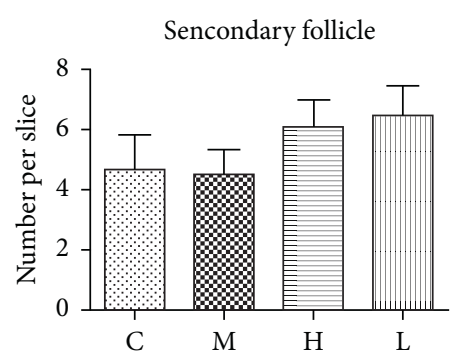

(c)

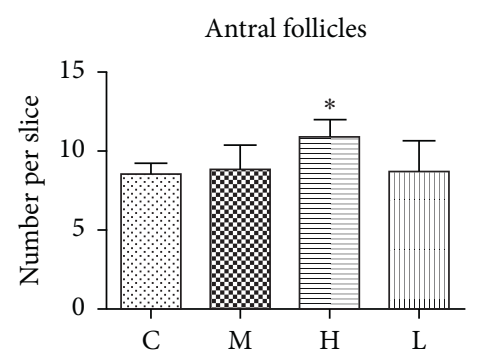

(d)

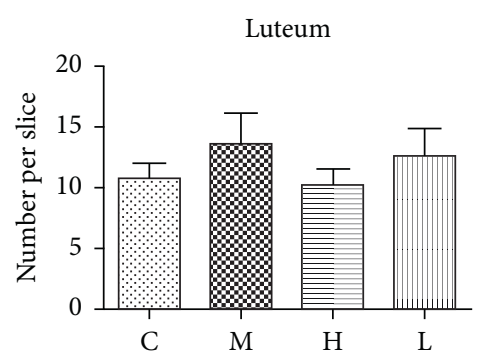

(e)

Figure 2: The number of follicles at different stages $(\mathrm{a}-\mathrm{d})$ and the number of corpus luteum (e) in the ovaries of rats in each group. *: significant compared with group $(\mathrm{C}) p<0.05$; \#: significant compared with group $(\mathrm{M}) p<0.05$.

compared to larger follicles; we did not observe any difference in the $E_{2}$ and $T$ level between each group (Figure 3).

\subsection{Effects of GNC on the Expression of Biomarkers of Ovarian} Reserve and Hormone Receptors in the Ovary. To better verify the effect of GNC on the improvement of ovarian reserve, we performed IHC and WB assays to determine the protein expression levels of several biomarkers of ovarian reserve. $\mathrm{MVH}$ is expressed in the cytoplasm of the oocyte in follicles at various stages and was mainly expressed in primordial follicles and primary follicles in this experiment. $\mathrm{AMH}$ is mainly expressed in the cytoplasm of secondary follicles and the granulosa cells of early antral follicles. As shown in Figures 4(a)-4(c), there were more deeply stained MVH and $\mathrm{AMH}$ positive follicles in group $\mathrm{C}$ and $\mathrm{H}$ than those in groups $\mathrm{M}$ and $\mathrm{L}(P<0.05)$. The intensity of DAB staining in primordial follicles in group L was slightly higher than that in group $\mathrm{M}$, and the differences were not significant. FSHR is the receptor of FSH and intermediated the promotion effects of FSH on follicle development. Similar results with MVH and AMH were found in FSHR expression in small and large secondary follicles and antral follicles, where FSHR was mainly expressed (Figures 4(a) and 4(d)). The group C and group $\mathrm{H}$ had higher expression of FSHR than that in the group $M(P<0.05)$. The group L showed less difference compared with the group $M$. Estrogen is another important regulatory factor in follicle development. ER $\alpha$ is mainly expressed in the nucleus of oocytes in follicles at various stages and stromal cells, while ER $\beta$ is mainly expressed in the cytoplasm of oocytes in follicles at various stages, especially in primordial follicles, primary follicles, and secondary follicles, with no expression in the nucleus [22]. There was no significant difference in the ER $\alpha$ expression level among follicles of ovaries at different stages in any group by immunohistochemistry (Figure 4(e)). But VCD induced a significantly downregulated $\operatorname{ER} \beta$ expression in ovaries (Figure $4(\mathrm{f}),(P<0.05))$. Also, high-dose of GNC significantly recovered the expression level of ER $\beta$ compared to the model group. There was also no significant effect of low-dose of GNC observed in regulating ER $\beta$ expression.

Western blot analysis confirmed most of the results of immunohistochemistry. As shown in Figures 5(a)-5(d), the expression of MVH, AMH, and FSHR protein in the group $\mathrm{M}$ was significantly lower than that in the group $\mathrm{C}$. The expression of $\mathrm{MVH}, \mathrm{AMH}$, and FSHR proteins was higher in the group $\mathrm{H}$ than that in the group $\mathrm{M}$ and was similar to that in the group C. The expression levels of $\mathrm{MVH}, \mathrm{AMH}$, and FSHR in the group L were also slightly increased or remained relatively low compared to the group $\mathrm{M}$. Although group L showed a decreased ER $\alpha$ compared to the group C, there was no significant difference between the groups $\mathrm{M}, \mathrm{H}$, and L (Figure 5(e)). ER $\beta$ showed similar trends between different groups as MVH, AMH, and FSHR. High-dose of GNC improved ER $\beta$ expression to a relatively normal level than the group M. Also, the group L tended to have a higher level of ER $\beta$ expression than that in the group $M$, but the difference was not significant (Figure 5(f)).

4.5. Effects of GNC on the PI3K-AKT Signaling Pathway. A previous report claimed that the inhibition of the PI3KAKT pathway intermediated the adverse effect of VCD on primordial and primary follicle atresia [17]. Therefore, we further explored the effect of GNC on the expression of upstream and key regulators of the PI3K-AKT signaling pathway and downstream apoptosis-related targets through western blot and immunohistochemistry. As shown in Figures 6(a) and 6(b), there was no significant difference in PTEN expression in ovarian tissues among different groups. 


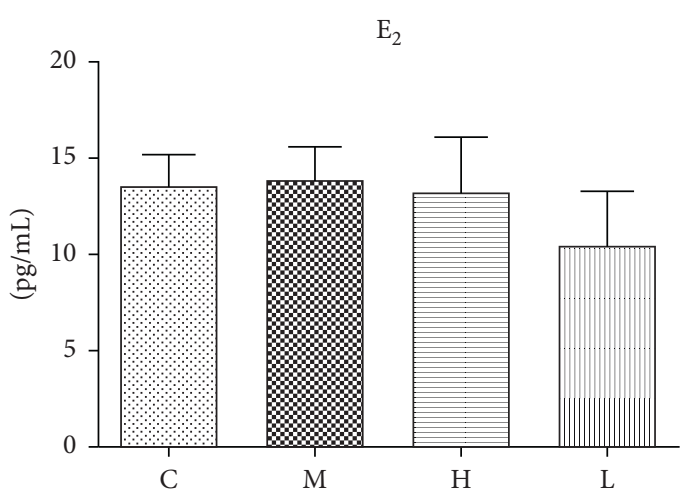

(a)

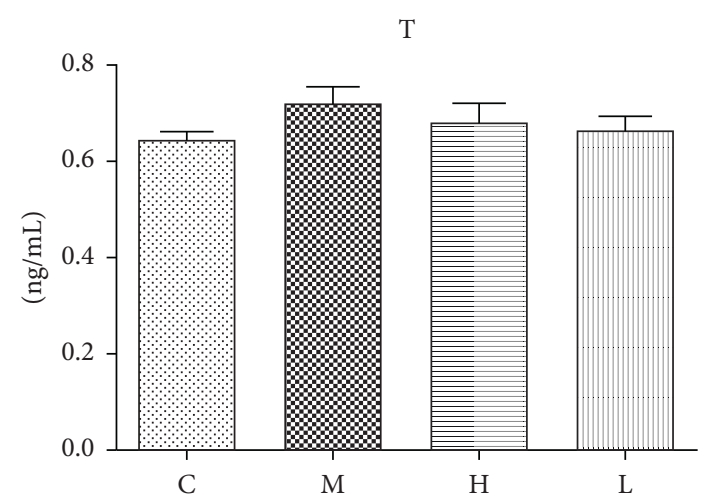

(b)

Figure 3: Serum $E_{2}$ (a) and $\mathrm{T}$ (b) levels among rats in different groups.

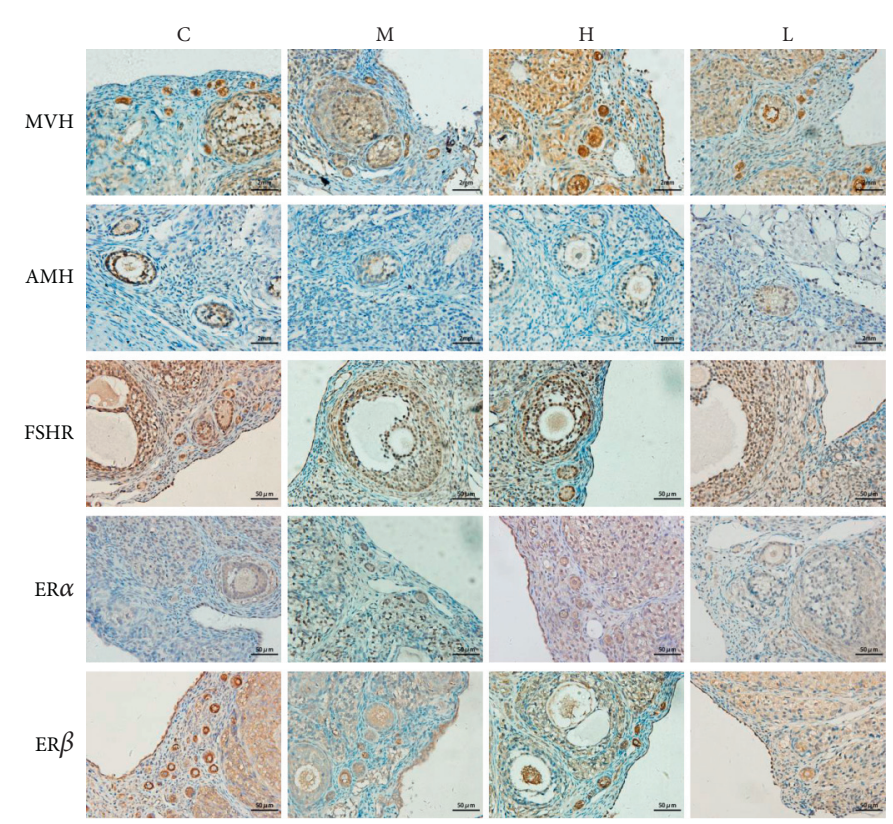

(a)

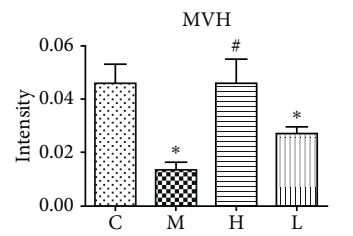

(b)

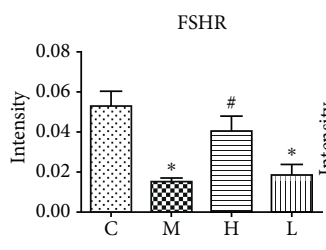

(d)

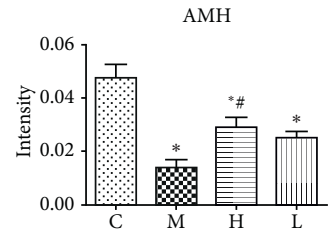

(c)

ER $\alpha$

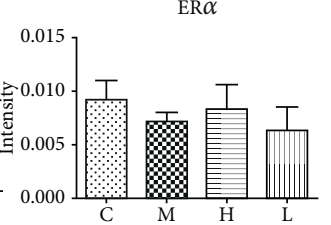

(e)

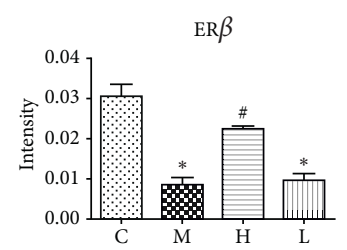

(f)

FIGURE 4: Immunohistochemical analysis of the expression of various biomarkers for ovarian reserve in each group. ${ }^{*}$ : significant compared with group $(C)(p<0.05)$; \#: significant compared with group $(M)(p<0.05)$.

Among the downstream signal molecules, the relative protein expression levels of $\mathrm{p}-\mathrm{AKT} / \mathrm{AKT}$ and FoxO3a in the group $\mathrm{M}$ were significantly decreased compared to that of the group C. Also, the group H showed a significantly higher expression of these proteins than that in the group M. The difference between the group $\mathrm{L}$ and group $\mathrm{M}$ was not significant (Figures 6(a)-6(d)). Besides, the expression of the proapoptotic proteins, Bad, Bax, cleaved caspase- 3 , and PARP was significantly increased in the group $M$ compared to the group C. Compared with the group $\mathrm{M}$, the levels of the above proapoptotic proteins in the group $\mathrm{H}$ were significantly decreased (Figures 6(e)-6(h)).
These changes in signaling molecules were further confirmed by immunohistochemistry analysis. As shown in Figure 7, among all the tested PI3K-AKT pathway molecules, VCD mainly induced a significant decrease of AKT phosphorylation (Figure $7(\mathrm{c}),(P<0.05))$ and a significant increase of BAX (Figure $7(\mathrm{e}),(P<0.05)$ ) and cleaved caspase-3 (Figure $7(\mathrm{f}),(P<0.05))$ expression in rat ovaries. High-dose of GNC significantly increased the AKT phosphorylation level and decreased the expression of BAX and cleaved caspase-3 (Figures 7(c), 7(e), and 7(f)). But lowdose of GNC did not show significant therapeutic effects on the regulation of the PI3K-AKT signaling pathway. 


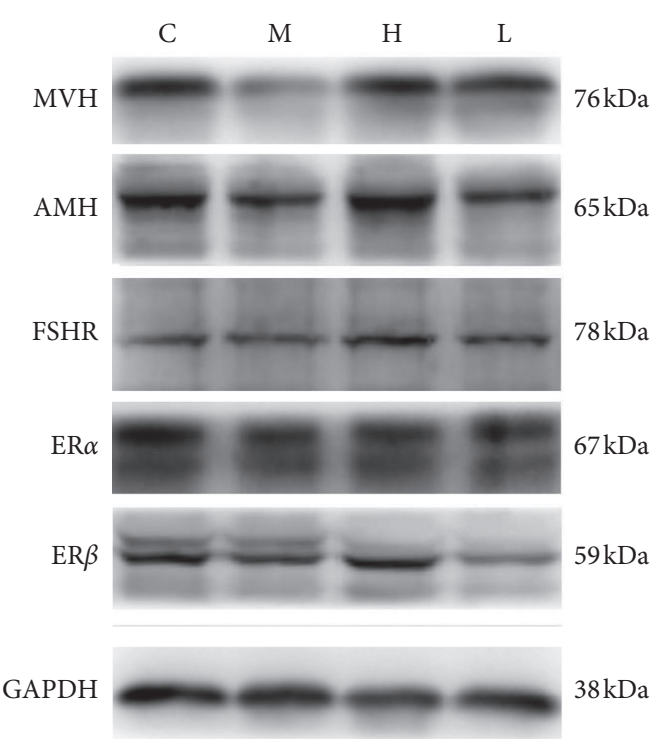

(a)

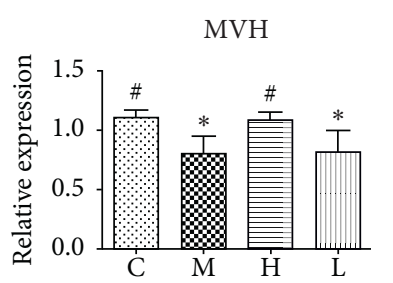

(b)

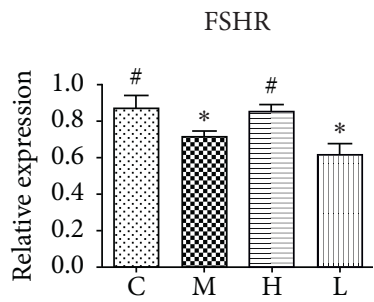

(d)

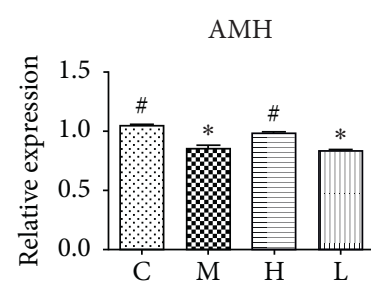

(c)

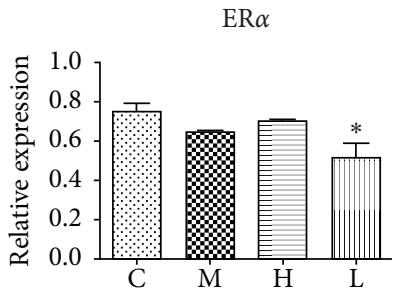

(e)

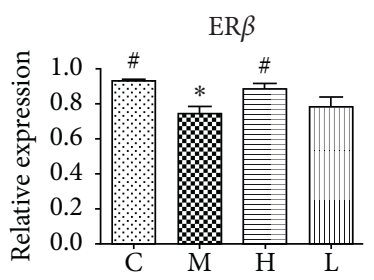

(f)

FIgURE 5: Western blot analysis of ovarian reserve function-related protein (MVH, AMH, and FSHR) and ER subtypes (ER $\alpha$ and ER $\beta$ ) in ovarian follicles of each group. *: significant compared to group $(\mathrm{C})(P<0.05)$; \#: significant compared to the group $(\mathrm{M})(P<0.05)$.

\section{Discussion}

DOR is one of the main causes of poor infertility outcomes and a major challenge even for patients who turned to assisted reproductive therapy for help. Although hormone replacement therapy used by Western medicine can ameliorate the clinical symptoms such as amenorrhea to some extent, the actual ovarian reserve is not fundamentally improved. Also, there exist side effects of reproductive hormone application. Traditional Chinese medicine has a special strength in treating female reproductive problems. As a long used multiherbal formula, GNC has been proved to be effective in treating a series of menopause and agingrelated diseases $[4,5,8,23]$. Based on preliminary clinical application and the speculation that DOR can be considered for the acceleration of ovarian reserve decline ahead of age, we brought up the assumption that GNC should be effective in treating DOR. In this study, by using a DOR rat model induced by VCD, we have validated that GNC is effective in protecting ovarian reserve by activating the AKT signaling pathway and decreased cellular apoptosis.

Decreased number and quality of available follicles are major characteristics of DOR. Both our present results (Figure 2) and a previous report [15] have confirmed that VCD can specifically induce the depletion of primordial and primary follicles without affecting other important organs [24]. This delicately replicates the initial stage of DOR and makes VCD a good agent to induce a DOR model than other approaches to establish DOR models like chemotherapy drugs. One of the important clinical abnormalities of DOR patients is the elevated serum FSH level. However, since VCD only influences follicle numbers of initial stages of follicle stages, the serum FSH level remains unaffected in model rats (data not shown). This may also explain the results that serum $E_{2}$ and $T$ levels remained unchanged in model rats compared to rats in the control group (Figure 3). But we found a significantly decreased FSHR protein expression in western blot and immunohistochemistry analysis (Figures 4(d) and 5(d)). This indicates a decreased ovarian response to FSH stimulation in model rats. MVH is a specific marker of germ cells expressed in the oocyte of follicles at various stages [25]. AMH is mainly secreted by granulosa cells of primary follicles, secondary follicles, and small antral follicles and has been widely applied in the clinic as a good marker to predict ovarian reserve because it does not fluctuate with the menstrual cycle [26]. We selected these two molecules as biomarkers of follicles at the early stages to validate the effects of VCD. Also, we found significantly downregulated $\mathrm{MVH}$ and $\mathrm{AMH}$ protein expression in the ovaries of model rats (Figures 4(b) and 4(c) and Figures 5(b) and $5(\mathrm{c})$ ), thus confirming the effectiveness of the VCDinduced DOR model.

Based on the model, the effects of GNC were revealed by the increased ovarian index and the increased number of primordial and primary follicles (Figures 2(a) and 2(b)). Also, surprisingly, the antral follicle count was also 

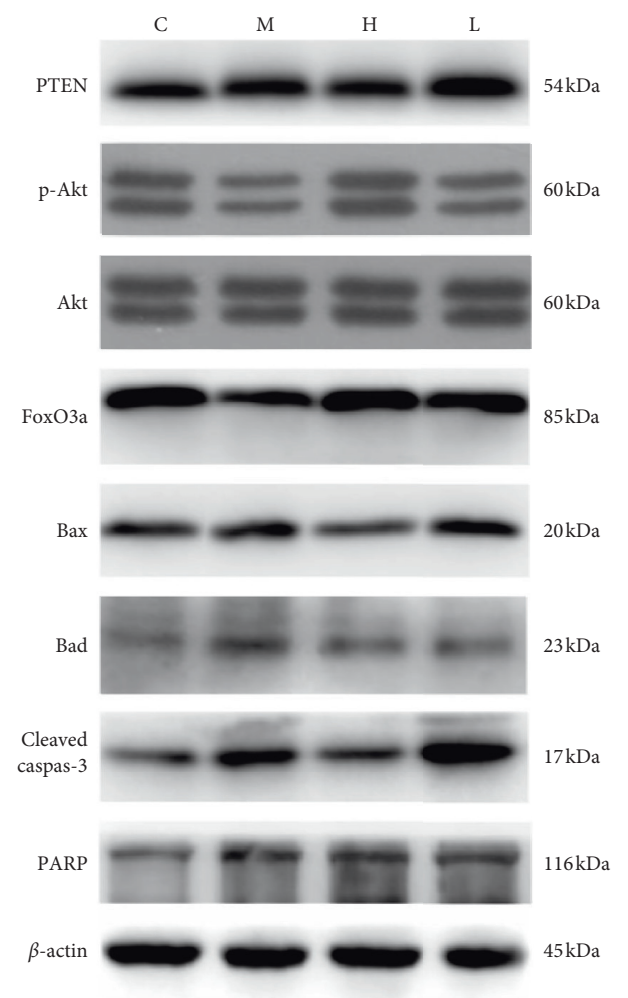

(a)

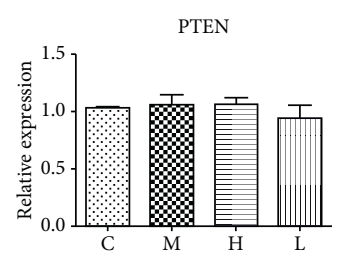

(b)

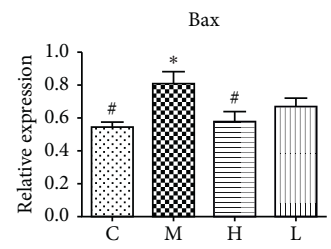

(e)

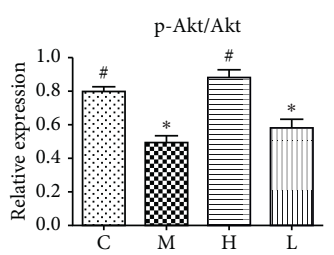

(c)

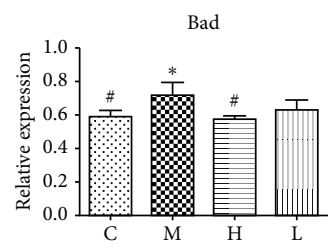

(f)

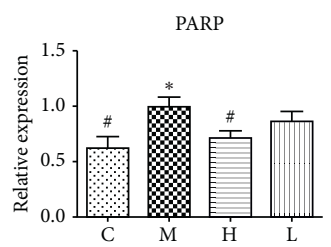

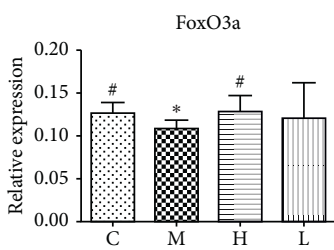

(d)

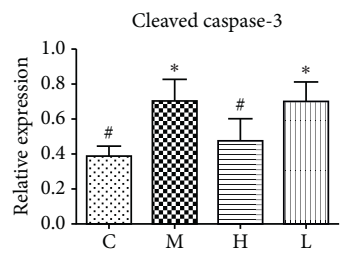

(g)

(h)

FIGURE 6: Western blot analysis of the protein expression of AKT signaling molecules in rat ovaries of each group. *: significant compared to group $(\mathrm{C})(P<0.05)$; \#: significant compared to group $(\mathrm{M})(P<0.05)$.

significantly improved compared to the control group by high-dose of GNC. This effect may indicate that GNC not only improves follicle development in the early stages but also promotes antral follicle development. $\mathrm{MVH}, \mathrm{AMH}$, and FSHR expression levels were also found to be increased as high as normal rats in high-dose of the GNC group. These results confirmed that GNC can improve the ovarian reserve of the VCD-induced DOR rat model and improve ovarian responsiveness to FSH stimulation, although only high-dose of GNC was found to be effective enough. Since traditional Chinese medicine puts much emphasis on the prevention of disease, we started GNC treatment at the same time as VCD administration. This raises two possibilities for the increase of primordial follicle and primary follicle numbers. The first possibility is that GNC prevented the damage caused by VCD, thus prevented the loss of follicles in the first place. This is the possibility we further validated. The second possibility is that GNC increased the formation of primordial and primary follicles after the VCD damage. Traditional dogma holds that the ovarian follicle pool is fixed after birth. The number of follicles will only decrease with the increase of age. However, recent studies have raised the possibility of the existence of ovarian germline stem cells $[27,28]$ or the formation of primordial follicles by bonemarrow-originated stem cells $[29,30]$. Also, the other traditional Chinese herbal medicine study has also found similar results of improved $\mathrm{MVH}$ expression, indicating the increase of primordial follicle numbers [31]. Whether the effects of GNC involve the participation of stem cells remains to be studied.

According to previous reports [17], VCD inhibits the phosphorylation and nuclear localization of AKT in the oocyte of primordial and small primary follicles. Also, one of the canonical effectors of FSHR is the PI3K-AKT pathway. To figure out whether GNC prevents this inhibition effect by improving FSHR-related AKT signaling activation and further reduced ovarian cell apoptosis, we analyzed the protein expression of several key targets of the PI3K-AKT signaling pathway by western blot and immunohistochemistry. We found that VCD significantly inhibited the p-AKT expression, decreased FOXO3a level, and increased ovarian proapoptosis molecules BAX, cleaved caspase- 3 , and PARP expression, while high-dose of GNC significantly promoted AKT phosphorylation, increased FOXO3a level, and decreased the above proapoptosis molecules (Figures 6 and 7). It is worth noticing that PI3K-AKT signaling is a critical signaling pathway in both granulosa cells and oocytes. In granulosa cells, it is related to cell proliferation, apoptosis, and steroidogenesis [32]. This AKT-activation effect is beneficial for both oocyte and granulosa cells to promote follicle maturation.

Except for gonadotrophins, estrogen is also an essential hormone for the regulation of follicle development. Cellular responses to estrogens are mediated by $\mathrm{ER} \alpha$ and $\mathrm{ER} \beta$. In this 


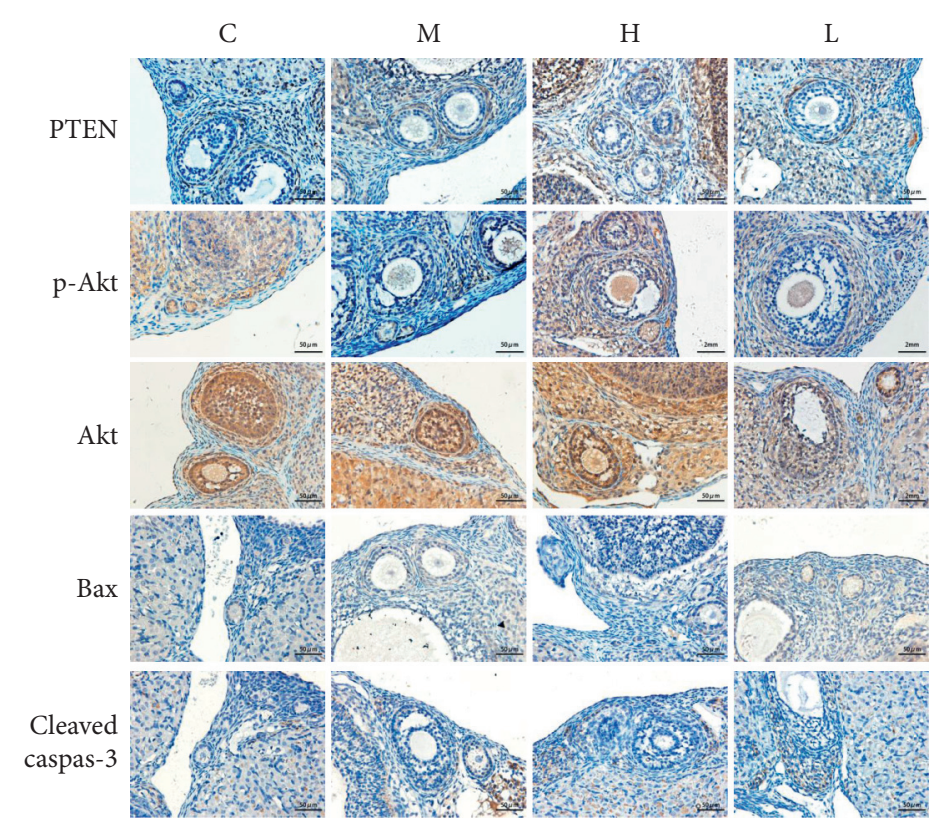

(a)

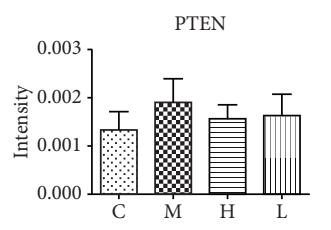

(b)

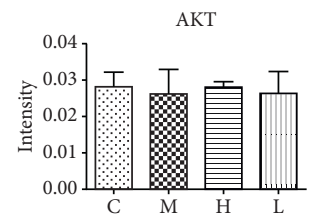

(d)

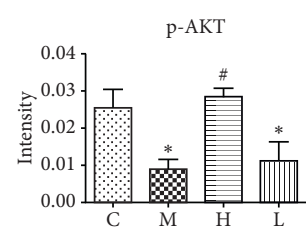

(c)

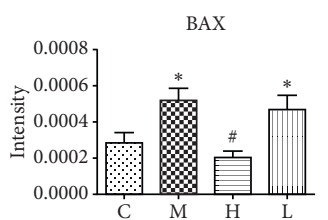

(e)

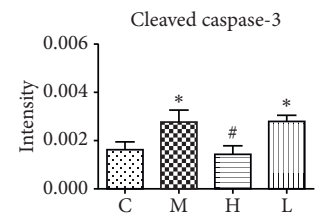

(f)

FIGURE 7: Immunohistochemistry analysis of AKT signaling pathway molecules in different groups. *: significant compared to group (C) $(P<0.05)$; \#: significant compared to group $(\mathrm{M})(P<0.05)$.

study, we found neither VCD nor GNC exerts a significant effect on ER $\alpha$ expression. But VCD significantly decreased $\mathrm{ER} \beta$ expression in the ovaries, and high-dose of GNC recovered ER $\beta$ protein level. This is consistent with our previous finding that GNC is capable of increasing the expression of $\mathrm{ER} \beta$ in the hypothalamus of ovariectomized rats [33]. As a nuclear receptor, it has been well recognized in an ER $\beta$ mutant rat model study that DNA binding-dependent transcriptional function of $\operatorname{ER} \beta$ is critical for preovulatory follicle maturation and ovulation [34]. But extranuclear signals including the PI3K-AKT pathway have been reported to be activated by $\operatorname{ER} \beta$ in brain disease studies $[35,36]$ and myocardial studies [37]. The effect of nontranscriptional activation of $\operatorname{ER} \beta$ remains unknown in ovarian researches. Therefore, whether the effect of AKT-activation involves the increased $\operatorname{ER} \beta$ activity requires further study.

In summary, GNC improves ovarian reserve in VCDinduced DOR rats. The underlying mechanism was possibly through the inhibition of cell apoptosis and primary follicle atresia through the activation of the AKT signaling pathway. Whether GNC acts on the AKT signaling pathway directly by stimulating FSHR or ER $\beta$ needs further verification by in vitro experiments.

\section{Data Availability}

All data used to support the findings of this study are available from the corresponding author upon request.

\section{Conflicts of Interest}

The authors declare that there are no conflicts of interest.

\section{Acknowledgments}

This work was supported by the National Natural Science Foundations of China (grant no. 81273956) and the Science and Technology Commission of Shanghai Municipality (grant no. 18401932000).

\section{References}

[1] F. I. Sharara, R. T. Scott Jr., and D. B. Seifer, "The detection of diminished ovarian reserve in infertile women," American Journal of Obstetrics and Gynecology, vol. 179, no. 3, pp. 804-812, 1998.

[2] R. Lew, "Natural history of ovarian function including assessment of ovarian reserve and premature ovarian failure," Best Practice \& Research Clinical Obstetrics \& Gynaecology, vol. 55, pp. 2-13, 2019.

[3] J. Cohen, N. Chabbert-Buffet, and E. Darai, "Diminished ovarian reserve, premature ovarian failure, poor ovarian responder-a plea for universal definitions," Journal of Assisted Reproduction and Genetics, vol. 32, no. 12, pp. 1709-1712, 2015.

[4] Y. Zhang, Y. Cao, and L. Wang, "The effects of a new, improved Chinese medicine, Gengnianchun formula granules, on hot flushes, depression, anxiety, and sleep in Chinese periand postmenopausal women: a randomized placebo-controlled trial," Menopause, vol. 27, no. 8, pp. 899-905, 2020.

[5] F. Meng, J. Li, Y. Rao et al., "Gengnianchun extends the lifespan of Caenorhabditis elegans via the insulin/IGF-1 signalling pathway," Oxidative Medicine and Cellular Longevity, vol. 2018, Article ID 4740739, 2018.

[6] P. L. Chen, W. J. Wang, Y. Q. Rao et al., "Serum containing Gengnianchun formula suppresses amyloid betainduced inflammatory cytokines in BV2 microglial cells by inhibiting the 
NFkappaB and JNK signaling pathways," Molecular Medicine Reports, vol. 17, no. 4, pp. 5043-5048, 2018.

[7] F. Meng, J. Li, W. Wang et al., "Gengnianchun, a traditional Chinese medicine, enhances oxidative stress resistance and lifespan in Caenorhabditis elegans by modulating daf-16/ FOXO," Evidence-Based Complementary and Alternative Medicine, vol. 2017, Article ID 8432306, 2017.

[8] F. Meng, J. Li, Y. Rao et al., "A Chinese herbal formula, gengnianchun, ameliorates beta-amyloid peptide toxicity in a Caenorhabditis elegans model of alzheimer's disease," Evidence-Based Complementary and Alternative Medicine, vol. 2017, Article ID 7480980, 2017.

[9] Y. Q. Rao, J. Li, and W. J. Wang, "Effects of Gengnianchun on learning and memory ability, neurotransmitter, cytokines, and leptin in ovariectomized rats," International Journal of Clinical and Experimental Medicine, vol. 8, no. 6, pp. 86488660, 2015.

[10] W. Wang, D. J. Li, J. Li et al., "An in vitro study on neuroprotective effects of serum containing Gengnianchun decoction and its main monomers against amyloid beta protein-induced cellular toxicity," Journal of Chinese Integrative Medicine, vol. 8, no. 1, pp. 67-73, 2010.

[11] J. Li, B. Li, W. J. Zhou et al., "Medicated rat serum containing Gengnianchun decoction reduces apoptosis of pheochromocytoma cells insulted by amyloid beta protein," Journal of Chinese Integrative Medicine, vol. 8, no. 5, pp. 472-479, 2010.

[12] F. G. Zhao, W. J. Wang, and W. J. Zhou, " [Effect of Gengnianchun Recipe on learning memory function and hippocampal cholinergic system in ovariectomized rats]," Zhongguo Zhong Xi Yi Jie He Za Zhi, vol. 28, no. 3, pp. 234-237, 2008.

[13] K. J. Liu, W. J. Wang, D. J. Li et al., "Effect of Gengnianchun Recipe on bone mineral density, bone biomechanical parameters and serum lipid level in ovariectomized rats," Chinese Journal of Integrative Medicine, vol. 12, no. 2, pp. 132-136, 2006.

[14] P. B. Hoyer, P. J. Devine, X. Xiaoming Hu, K. E. Thompson, and I. G. Sipes, "Ovarian toxicity of 4-vinylcyclohexene diepoxide: a mechanistic model," Toxicologic Pathology, vol. 29, no. 1, pp. 91-99, 2001.

[15] F. Zhao, Y. Lian, and W. Wang, "Short-term effects of 4vinylcyclohexene diepoxide on ovarian follicular depletion and fertility in Sprague-Dawley rats," International Journal of Clinical and Experimental Medicine, vol. 10, no. 11, pp. 15775-15781, 2017.

[16] Z. Chen, X. Kang, L. Wang et al., "Rictor/mTORC2 pathway in oocytes regulates folliculogenesis, and its inactivation causes premature ovarian failure," Journal of Biological Chemistry, vol. 290, no. 10, pp. 6387-6396, 2015.

[17] A. F. Keating, S. M. Fernandez, C. J. Mark-Kappeler, N. Sen, I. G. Sipes, and P. B. Hoyer, "Inhibition of PIK3 signaling pathway members by the ovotoxicant 4-vinylcyclohexene diepoxide in rats," Biology of Reproduction, vol. 84, no. 4, pp. 743-751, 2011.

[18] X. Xing, L. Su, P. F. Asare et al., "Danzhi Qing'e (DZQE) activates AMPK pathway and regulates lipid metabolism in a rat model of perimenopausal hyperlipidaemia," Experimental Physiology, vol. 101, no. 11, pp. 1406-1417, 2016.

[19] S. Y. Xu, R. L. Bian, and X. Chen, Pharmacological Experimental Methods. 3, People's Medical Publishing House, Beijing, China, 2002.

[20] E. Helen Turner and T. Vivien, "Principles and practice of endocrinology and metabolism: 3rd edition on CD-ROM," Clinical Endocrinology, vol. 59, no. 5, p. 655, 2003.
[21] K. Oktay, R. S. Schenken, and J. F. Nelson, "Proliferating cell nuclear antigen marks the initiation of follicular growth in the rat1," Biology of Reproduction, vol. 53, no. 2, pp. 295-301, 1995.

[22] M. Sar and F. Welsch, "Differential expression of estrogen receptor- $\beta$ and estrogen receptor- $\alpha$ in the rat ovary," Endocrinology, vol. 140, no. 2, pp. 963-971, 1999.

[23] J. Li, W. J. Wang, D. J. Li et al., "Gengnianchun recipe inhibits apoptosis of pheochromocytoma cells from beta-amyloid 2535 insult, better than monotherapies and their compounds," Neural Regeneration Research, vol. 6, no. 36, pp. 2815-2821, 2011.

[24] C. J. Kappeler and P. B. Hoyer, "4-vinylcyclohexene diepoxide: a model chemical for ovotoxicity," Systems Biology in Reproductive Medicine, vol. 58, no. 1, pp. 57-62, 2012.

[25] Z. Pan, M. Sun, J. Li et al., "The expression of markers related to ovarian germline stem cells in the mouse ovarian surface epithelium and the correlation with notch signaling pathway," Cellular Physiology and Biochemistry, vol. 37, no. 6, pp. 2311-2322, 2015.

[26] A. La Marca, G. Sighinolfi, D. Radi et al., "Anti-Mullerian hormone $(\mathrm{AMH})$ as a predictive marker in assisted reproductive technology (ART)," Human Reproduction Update, vol. 16, no. 2, pp. 113-130, 2010.

[27] Y. A. R. White, D. C. Woods, Y. Takai, O. Ishihara, H. Seki, and J. L. Tilly, "Oocyte formation by mitotically active germ cells purified from ovaries of reproductive-age women," Nature Medicine, vol. 18, no. 3, pp. 413-421, 2012.

[28] K. Zou, Z. Yuan, Z. Yang et al., "Production of offspring from a germline stem cell line derived from neonatal ovaries," Nature Cell Biology, vol. 11, no. 5, pp. 631-636, 2009.

[29] I. Virant-Klun, N. Zech, P. Rožman et al., "Putative stem cells with an embryonic character isolated from the ovarian surface epithelium of women with no naturally present follicles and oocytes," Differentiation, vol. 76, no. 8, pp. 843-856, 2008.

[30] I. Virant-Klun, "Very small embryonic-like stem cells: a potential developmental link between germinal lineage and hematopoiesis in humans," Stem Cells and Development, vol. 25, no. 2, pp. 101-113, 2016.

[31] K.-k. Song, W.-w. Ma, C. Huang et al., "Effect and mechanism of Bushen Huoxue recipe on ovarian reserve in mice with premature ovarian failure," Journal of Huazhong University of Science and Technology [Medical Sciences], vol. 36, no. 4, pp. 571-575, 2016.

[32] L. X. Chen and P. T. Jimenez, "Female fertility: it takes two to tango," Endocrinology, vol. 158, no. 7, pp. 2074-2076, 2017.

[33] F. G. Zhao, W. J. Wang, W. J. Zhou et al., "Regulation of learning and memory ability and hippocampal neuron ER subtype in ovariectomized rats by Gengnianchun Decotion," Shanghai Zhogn Yi Yao Za Zhi, vol. 41, no. 11, pp. 66-69, 2007.

[34] M. A. K. Rumi, P. Singh, K. F. Roby et al., "Defining the role of estrogen receptor $\beta$ in the regulation of female fertility," Endocrinology, vol. 158, no. 7, pp. 2330-2343, 2017.

[35] K. Nakaso, N. Tajima, Y. Horikoshi et al., "The estrogen receptor $\beta$-PI3K/Akt pathway mediates the cytoprotective effects of tocotrienol in a cellular Parkinson's disease model," Biochimica et Biophysica Acta (BBA)-Molecular Basis of Disease, vol. 1842, no. 9, pp. 1303-1312, 2014.

[36] K. L. Wu, C.-H. Chen, and C.-D. Shih, "Nontranscriptional activation of PI3K/Akt signaling mediates hypotensive effect following activation of estrogen receptor $\beta$ in the rostral 
ventrolateral medulla of rats," Journal of Biomedical Science, vol. 19, no. 1, p. 76, 2012.

[37] M. Wang, Y. Wang, B. Weil et al., "Estrogen receptor $\beta$ mediates increased activation of PI3K/Akt signaling and improved myocardial function in female hearts following acute ischemia," American Journal of Physiology-Regulatory, Integrative and Comparative Physiology, vol. 296, no. 4, pp. R972-R978, 2009. 